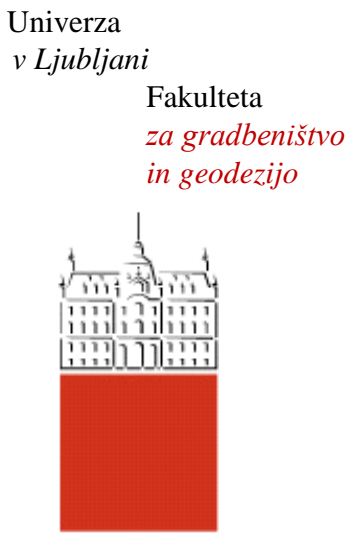

Jamova cesta 2

1000 Ljubljana, Slovenija

http://www3.fgg.uni-lj.si/

\section{DRUGG - Digitalni repozitorij UL FGG http://drugg.fgg.uni-lj.si/}

Ta članek je avtorjeva zadnja recenzirana različica, kot je bila sprejeta po opravljeni recenziji.

Prosimo, da se pri navajanju sklicujte na bibliografske podatke, kot je navedeno:
University
of Ljubljana

Faculty of Civil and Geodetic Engineering

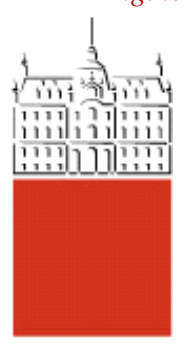

Jamova cesta 2 SI - 1000 Ljubljana, Slovenia http://www3.fgg.uni-lj.si/en/

DRUGG - The Digital Repository http://drugg.fgg.uni-lj.si/

This version of the article is author's manuscript as accepted for publishing after the review process.

When citing, please refer to the publisher's bibliographic information as follows:

Zupan, D., Saje, M. 2004. Rotational invariants in finite element formulation of threedimensional beam theories. Computers \& Structures 82: 2027-2040. DOI: 10.1016/j.compstruc.2004.03.069. 


\title{
Rotational invariants in finite element formulation of three-dimensional beam theories
}

\author{
D. Zupan, M. Saje* \\ University of Ljubljana, Faculty of Civil and Geodetic Engineering, Jamova 2, \\ SI-1115 Ljubljana, Slovenia
}

\begin{abstract}
This paper introduces a new finite element formulation of the 'geometrically exact finite-strain beam theory'. The formulation employs the generalized virtual work principle. In the resulting governing equations of the beam, the strain vectors are the only unknown functions. The consistency condition that the equilibrium and the constitutive internal force and moment vectors are equal, is enforced to be satisfied at chosen points. The accuracy and the efficiency of the derived numerical algorithm are demonstrated by several examples.
\end{abstract}

Key words: non-linear beam theory, finite element method, three-dimensional rotation, rotational invariant, strain measure, invariant preserving algorithm

\section{Introduction}

In the paper we limit ourselves to the three-dimensional beam model, derived from the resultant form of the differential equilibrium equations. The model, often called the 'geometrically exact finite-strain beam theory' (Reissner [14], Simo [17]), introduces six strain measures: a longitudinal strain, two shear strains, and three pseudo-curvatures.

The geometry of the three-dimensional beam is described by the line of centroids of cross-sections and by the family of the cross-sections not necessarily normal to the line of centroids at deformed state; therefore, the configuration

* Corresponding author. Tel.: +38614768 613; FAX: +38614768629 Email address: msaje@fgg.uni-lj.si (M. Saje). 
space of the beam consists of (i) the linear space of position vector of the line of centroids, and (ii) the non-linear space of rotations of cross-sections. Because the spatial rotations are elements of the non-linear space, the configuration space of the beam is a non-linear manifold. That is what makes the problem of three-dimensional beams so demanding.

The essential part of any formulation is the choice of primary variables. Earlier formulations base their finite element implementation on both displacements and rotations as the interpolated degrees of freedom (as, e.g., Simo and VuQuoc [18], Ibrahimbegovic [8], Crisfield [5]), or solely on rotations (Jelenić and Saje [10]). In these approaches, the rotations or/and their increments are interpolated in a standard additive manner, neglecting the fact that the rotations are physically non-additive quantities. As reported by Crisfield and Jelenić [6], such an approach leads to non-objective discrete strain measures. Crisfield and Jelenić [6] suggest a strain-objective formulation, requiring a construction of special interpolating functions, which is a complicated task for higher-order finite elements.

In contrast to the above mentioned formulations, we here assume that the total strain measures, i.e. total membrane and shear strains and the total pseudo-curvatures, are primary interpolated variables. In order to apply the strain measures as basic variables, we follow the work by Planinc et al. [13] and extend it to three-dimensional beams and frames by proposing a modified principle of virtual work, in which only the strain vectors need to be interpolated. Recently we have proposed a formulation (Zupan and Saje [24]) in which the only primary interpolated variables are the variations (increments) of pseudo-curvatures (the variations of membrane and shear strains are not interpolated). This 'one field' formulation proves to be highly accurate and computationally economic when an elastic material model can be assumed.

The integration of rotations from the given interpolated total pseudo-curvatures seems to be the fundamental problem of such a formulation. In the planar case, the derivative of rotations with respect to the natural parameter of the line of centroids equals the pseudo-curvature. In three dimensions, the derivative of rotations equals the product of a rotation-dependent transformation matrix and the rotational strain. Such a system of differential equations cannot be integrated analytically. That is why, in three-dimensional beam theories, the rotational strain is very rarely chosen as a primary variable in the numerical formulation. Tabarrok et al. [21] assumed an analytically integrable curvature distribution in order to develop a more suitable interpolation for displacements and rotations. Schulz and Filippou [15] interpolated both the infinitesimal (incremental) curvatures and the infinitesimal rotations; in their formulation, the objectivity of the total rotations and the total curvatures may be questioned.

In a sharp contrast to the above cited authors, our solution employs the exact relationship between the total rotational strains (here also called pseudo- 


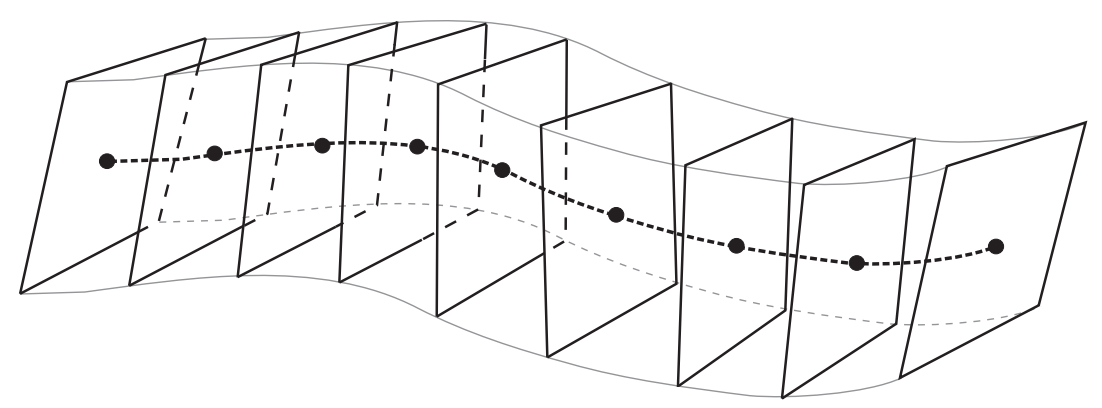

Fig. 1. Model of the three-dimensional beam: a curve and a family of planes.

curvatures) and the rotations. Due to the complicated form of the kinematical equations, the numerical method is used for the integration of the total rotations from the given total rotational strains. The linearity of the space of basic variables - the strains - simplifies Newton's iteration method when compared to the case where the rotations are basic variables, and leads automatically to the objectivity of the interpolated strains.

In standard finite-element formulations the stress-resultants, obtained from the equilibrium equations, and those found from the constitutive equations, are not equal. In the present formulation this 'inconsistency of equilibrium at cross-sections' is resolved by enforcing the consistency condition to be satisfied in a set of predefined points (here taken to coincide with the interpolation points). A similar strategy was employed by Vratanar and Saje [23] for an elastic-plastic analysis of plane frames.

\section{Geometry and kinematics of the three-dimensional beam}

The geometry of the three-dimensional beam is described by the family of the plane cross-sections and by the line of centroids of cross-sections (Figure 1). According to the Bernoulli hypothesis we assume that a plane cross-section suffers only a rigid rotation during deformation. Note that the cross-sections are not necessarily normal to the line of centroids at their intersections. This makes it possible to include the effect of shear stresses into the beam analysis. The line of centroids represents a spatial curve and the cross-sections describe a family of planes in the three-dimensional space. For this abstract model, a suitable parametrization needs to be employed.

The line of centroids is described by the position vector $\vec{r}$ (see Figure 2). In order to describe the position of the cross-sections in space, a family of orthonormal vector bases $\left\{\vec{G}_{1}, \vec{G}_{2}, \vec{G}_{3}\right\}$ is introduced. Vectors $\vec{G}_{2}$ and $\vec{G}_{3}$ are directed along the principal axes of inertia of the particular cross-section, and 
$\vec{G}_{1}$ is normal to the cross-section: $\vec{G}_{1}=\vec{G}_{2} \times \vec{G}_{3}$. As the rotation of the crosssections varies along the beam, the basis $\left\{\vec{G}_{1}, \vec{G}_{2}, \vec{G}_{3}\right\}$ is different at each material point of the line of centroids; therefore, it is here called the "material basis'. It is found convenient to distinguish between the reference configuration, where the geometry and the deformations of the beam are known, and an arbitrary deformed configuration. That way we can express the vectors $\vec{r}$, $\vec{G}_{1}, \vec{G}_{2}$, and $\vec{G}_{3}$ as functions of parameter $x$, the arc-length of the reference line of centroids of cross-sections.

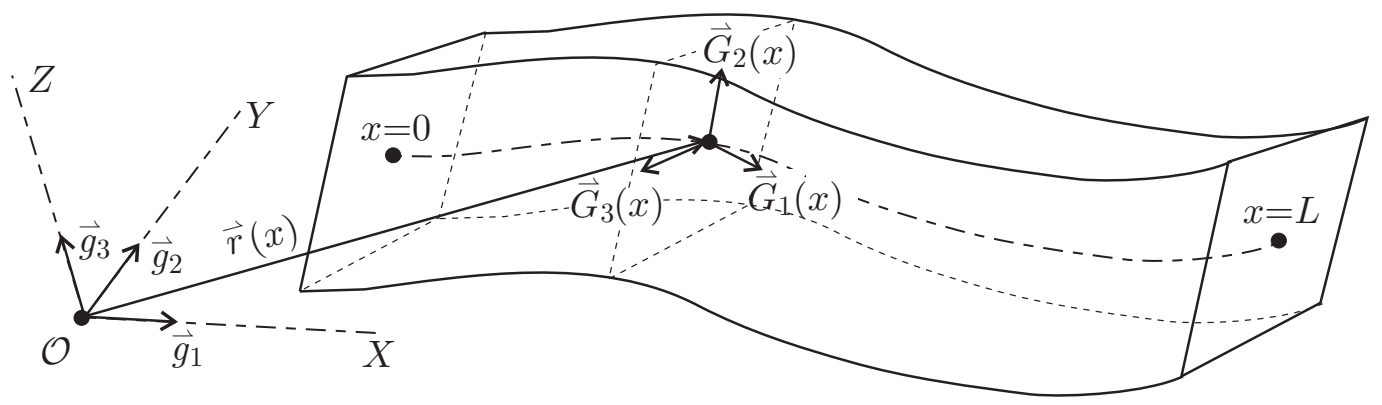

Fig. 2. Parametrization of the three-dimensional beam in the abstract vector space.

In order to describe conveniently the rotations of the cross-sections, an (arbitrary) reference point $\mathcal{O}$ and a triad of orthonormal base vectors $\left\{\vec{g}_{1}, \vec{g}_{2}, \vec{g}_{3}\right\}$ are introduced. The triad $\left\{\vec{g}_{1}, \vec{g}_{2}, \vec{g}_{3}\right\}$ is not related to the deformation of the beam and is henceforth referred to as the 'spatial basis'. Along with a reference point $\mathcal{O}$, the basis $\left\{\vec{g}_{1}, \vec{g}_{2}, \vec{g}_{3}\right\}$ defines the spatial Cartesian coordinate system $(X, Y, Z)$. The two bases are related by the rotational operator $\mathcal{R}(x)$, which maps $\left\{\vec{g}_{1}, \vec{g}_{2}, \vec{g}_{3}\right\}$ into $\left\{\vec{G}_{1}(x), \vec{G}_{2}(x), \vec{G}_{3}(x)\right\}$. Mathematically operator $\mathcal{R}(x)$ is a linear operator on the abstract three-dimensional Euclidean space, while it represents the physical rotation between the bases. The physical rotation in the three-dimensional space can be described by the axis of rotation and by the rotation angle; the axis and the rotation angle can be described by one vector, the rotational vector $\vec{\vartheta}[1]$. This vector is very suitable for the parametrization of the three-dimensional rotation. The rotational operator $\mathcal{R}$ is dependent directly on the rotational vector, and the relationship between the two quantities is referred to as the Rodrigues formula:

$$
\mathcal{R} \vec{r}=\vec{r}+\frac{\sin \vartheta}{\vartheta} \vec{\vartheta} \times \vec{r}+\frac{1-\cos \vartheta}{\vartheta^{2}} \vec{\vartheta} \times(\vec{\vartheta} \times \vec{r}) .
$$

$\vec{r}$ denotes an arbitrary vector and ' $x$ ' is the cross vector product.

In the derivation of the beam governing equations, the abstract vectors are often expressed by three scalar components with respect to the suitable or- 
thonormal basis. Yet we do not need to be restricted by the choice of the basis in the development of the governing equations. Therefore an abstract approach is shown here, using the algebra of abstract three-dimensional vectors.

\section{Strain measures and stress resultants}

We consider a beam subjected to the external distributed force and moment vectors $\vec{n}$ and $\vec{m}$ per unit length of the reference line of centroids. The stressresultant force vector over the cross-section is denoted by $\vec{N}$ and the resulting moment vector by $\vec{M}$. The equilibrium equations of an infinitesimal element of a beam are given by the following differential equations:

$$
\begin{aligned}
& \vec{n}(x)=-\vec{N}^{\prime}(x), \\
& \vec{m}(x)=-\vec{M}^{\prime}(x)-\vec{r}^{\prime}(x) \times \vec{N}(x) .
\end{aligned}
$$

The prime $\left({ }^{\prime}\right)$ denotes the derivative with respect to arc-length parameter $x$.

Following the approach of Reissner [14] and Simo [17], we introduce the strain vectors $\vec{\gamma}$ and $\vec{\kappa}$ which are consistent with the virtual work principle for an arbitrary part of the beam bounded by the cross-sections at $x=x_{1}$ and $x=x_{2}$ :

$$
\begin{aligned}
\int_{x_{1}}^{x_{2}} \vec{N} \cdot \delta(\vec{\gamma})_{r e l} d x+\int_{x_{1}}^{x_{2}} \vec{M} \cdot \delta & (\vec{\kappa})_{r e l} d x=\int_{x_{1}}^{x_{2}} \vec{n} \cdot \delta \vec{r} d x \\
& +\int_{x_{1}}^{x_{2}} \vec{m} \cdot \delta \vec{\vartheta} d x+[\vec{N} \cdot \delta \vec{r}+\vec{M} \cdot \delta \vec{\vartheta}]_{x_{1}}^{x_{2}}
\end{aligned}
$$

Note that the virtual work principle is written in an abstract vector form, in which the relative variation of strain vectors is introduced. The relative variation of a vector is a partial variation of a vector, in which the variation of rotations is disregarded : $\delta(\vec{a})_{\text {rel }}=\delta \vec{a}-\delta \vec{\vartheta} \times \vec{a}$. If the vector were, e.g., expressed with respect to the material basis, we would vary its components only and disregard the variation of the base vectors. This is in accord with the notion of 'objective rates'; see, e.g. Simo [17].

Inserting equations (2)-(3) into (4) and applying the partial integration yields the relationships between the variations of kinematic vector variables $(\vec{r}, \vec{\vartheta})$ and the relative variations of strain vectors $(\vec{\gamma}, \vec{\kappa})$

$$
\begin{aligned}
& \delta(\vec{\gamma})_{r e l}=\delta \vec{r}^{\prime}-\delta \vec{\vartheta} \times \vec{r}^{\prime} \\
& \delta(\vec{\kappa})_{r e l}=\delta \vec{\vartheta}^{\prime} .
\end{aligned}
$$


Equations (5) and (6) relate the variations of strains, displacements and rotations, and indicate that the variations of the quantities mentioned above are not all independent. Following the approach similar to that of Reissner [14], we can show that equations (5) and (6) can be integrated for strain measures $(\vec{\gamma}, \vec{\kappa})$ as functions of displacements and rotations $(\vec{r}, \vec{\vartheta})$, resulting in

$$
\begin{aligned}
& \vec{\gamma}=\vec{r}^{\prime}+\vec{c} \\
& \vec{\kappa}=\mathcal{T} \vec{\vartheta}^{\prime}+\vec{d} .
\end{aligned}
$$

Vector functions $\vec{c}$ and $\vec{d}$ are variational constants $\left(\delta(\vec{c})_{r e l}=\delta(\vec{d})_{r e l}=\overrightarrow{0}\right)$ to be determined from the known strains and kinematics of the beam at the reference configuration. The proof of the solution in (8) is beyond the scope of this article and is therefore omitted here (see, e.g., [1], [3], [7]). $\mathcal{T}$ is a linear operator connecting the derivative of the rotational vector, $\vartheta$, and the curvature vector, $\vec{\kappa}$, given by the vector equation

$$
\mathcal{T} \vec{\vartheta}^{\prime}=\vec{\vartheta}^{\prime}+\frac{1-\cos \vartheta}{\vartheta^{2}} \vec{\vartheta} \times \vec{\vartheta}^{\prime}+\frac{\vartheta-\sin \vartheta}{\vartheta^{3}} \vec{\vartheta} \times\left(\vec{\vartheta} \times \vec{\vartheta}^{\prime}\right)
$$

In contrast, result (7) can be easily proven. Considering that $\delta \vec{r}^{\prime}-\delta \vec{\vartheta} \times \vec{r}^{\prime}$ equals the relative variation of vector $\vec{r}^{\prime}$, equation (5) yields

$$
\delta(\vec{\gamma})_{r e l}=\delta\left(\vec{r}^{\prime}\right)_{r e l}
$$

from which it is apparent that $\vec{\gamma}$ and $\vec{r}^{\prime}$ differ only in the variational constant $\vec{c}$. From (9) it also follows that

$$
\delta(\vec{c})_{\text {rel }}=\overrightarrow{0}
$$

The equation says that if $\vec{c}$ is expressed with respect to the material basis, its components remain the same during the deformation, because their relative variations equal zero. Note, however, that $\vec{c}$ is still an arbitrary function of $x$. The same holds for vector $\vec{d}$.

We assume that the stress-resultant vectors, $\vec{N}$ and $\vec{M}$, directly depend on strains $\vec{\gamma}$ and $\vec{\kappa}$. Since we do not wish to restrict our analysis to a particular class of materials of the beam, a rather general form of constitutive equations is assumed

$$
\begin{aligned}
\vec{N} & =\mathcal{C}_{N}\left(\vec{\gamma}-\vec{\gamma}_{0}, \vec{\kappa}-\vec{\kappa}_{0}\right) \\
\vec{M} & =\mathcal{C}_{M}\left(\vec{\gamma}-\vec{\gamma}_{0}, \vec{\kappa}-\vec{\kappa}_{0}\right)
\end{aligned}
$$


with operators $\mathcal{C}_{N}$ and $\mathcal{C}_{M}$ being invariant under superimposed rigid-body motions and at least once differentiable with respect to $\vec{\gamma}, \vec{\kappa}$, and $x$, but otherwise arbitrary. $\vec{\gamma}_{0}$ and $\vec{\kappa}_{0}$ are strain vectors of the reference configuration. Consequently, the initial internal forces and moments need not to be zero.

\section{Generalized virtual work principle}

Rewriting the virtual work principle (4) for a beam of initial length $L$ gives

$$
\begin{aligned}
\int_{0}^{L}\left(\vec{N} \cdot \delta(\vec{\gamma})_{r e l}+\vec{M} \cdot \delta(\vec{\kappa})_{r e l}\right) d x=\int_{0}^{L}(\vec{n} \cdot \delta \vec{r}+\vec{m} \cdot \delta \vec{\vartheta}) d x \\
+\vec{S}^{0} \cdot \delta \vec{r}(0)+\vec{P}^{0} \cdot \delta \vec{\vartheta}(0)+\vec{S}^{L} \cdot \delta \vec{r}(L)+\vec{P}^{L} \cdot \delta \vec{\vartheta}(L)
\end{aligned}
$$

$\vec{S}^{0}, \vec{P}^{0}, \vec{S}^{L}, \vec{P}^{L}$ are vectors of the external point loads at the boundaries $x=0$ and $x=L \cdot \delta \vec{r}(0), \delta \vec{\vartheta}(0), \delta \vec{r}(L)$ and $\delta \vec{\vartheta}(L)$ are variations of the position vector and the rotational vector at $x=0$ and $x=L$.

In (12), the strain and the kinematic vectors are related by the kinematic conditions (7)-(8). According to the method of Lagrangian multipliers in constrained problems of calculus of variations and the related work of Planinc et al. [13] on planar beams, the constraining kinematic equations

$$
\begin{gathered}
\vec{\gamma}-\vec{r}^{\prime}-\vec{c}=\overrightarrow{0} \\
\vec{\kappa}-\mathcal{T} \vec{\vartheta}^{\prime}-\vec{d}=\overrightarrow{0}
\end{gathered}
$$

are scalarly multiplied by arbitrary, independent, at least once differentiable vector functions $\vec{a}(x)$ and $\vec{b}(x)$. The scalar products of the multipliers and the constraining equations are integrated along the length

$$
\begin{array}{r}
\int_{0}^{L} \vec{a} \cdot\left(\vec{\gamma}-\vec{r}^{\prime}-\vec{c}\right) d x=0 \\
\int_{0}^{L} \vec{b} \cdot\left(\vec{\kappa}-\mathcal{T} \vec{\vartheta}^{\prime}-\vec{d}\right) d x=0
\end{array}
$$

and varied with respect to $\vec{a}, \vec{b}, \vec{\gamma}, \vec{\kappa}, \vec{r}$, and $\vec{\vartheta}$

$$
\begin{array}{r}
\int_{0}^{L} \delta \vec{a} \cdot\left(\vec{\gamma}-\vec{r}^{\prime}-\vec{c}\right) d x+\int_{0}^{L} \vec{a} \cdot \delta\left(\vec{\gamma}-\vec{r}^{\prime}-\vec{c}\right) d x=0 \\
\int_{0}^{L} \delta \vec{b} \cdot\left(\vec{\kappa}-\overrightarrow{\mathcal{T}}^{\prime}-\vec{d}\right) d x+\int_{0}^{L} \vec{b} \cdot \delta\left(\vec{\kappa}-\overrightarrow{\mathcal{T}}^{\prime}-\vec{d}\right) d x=0 .
\end{array}
$$

The variations in the second integrals are the variations of equations (7) and (8). Their variational forms are given by equations (5) and (6). Thus, we can 
write

$$
\begin{array}{r}
\int_{0}^{L} \delta \vec{a} \cdot\left(\vec{\gamma}-\vec{r}^{\prime}-\vec{c}\right) d x+\int_{0}^{L} \vec{a} \cdot\left(\delta(\vec{\gamma})_{r e l}-\delta \vec{r}^{\prime}-\delta \vec{\vartheta} \times \vec{r}^{\prime}\right) d x=0 \\
\int_{0}^{L} \delta \vec{b} \cdot\left(\vec{\kappa}-\mathcal{T}^{\prime}{ }^{\prime}-\vec{d}\right) d x+\int_{0}^{L} \vec{b} \cdot\left(\delta(\vec{\kappa})_{r e l}-\delta \vec{\vartheta}^{\prime}\right) d x=0
\end{array}
$$

The terms $\vec{a} \cdot \delta \vec{r}^{\prime}$ and $\vec{b} \cdot \delta \vec{\vartheta}^{\prime}$ are partially integrated and the equality $\vec{a}$. $\left(\delta \vec{\vartheta} \times \vec{r}^{\prime}\right)=-\delta \vec{\vartheta} \cdot\left(\vec{a} \times \vec{r}^{\prime}\right)$ is employed. Then we obtain

$$
\begin{aligned}
\int_{0}^{L} \delta \vec{a} \cdot\left(\vec{\gamma}-\vec{r}^{\prime}-\vec{c}\right) d x+\int_{0}^{L} \vec{a} \cdot \delta & (\vec{\gamma})_{r e l} d x-\int_{0}^{L} \delta \vec{\vartheta} \cdot\left(\vec{a} \times \vec{r}^{\prime}\right) d x \\
& -[\vec{a} \cdot \delta \vec{r}]_{0}^{L}+\int_{0}^{L} \vec{a}^{\prime} \cdot \delta \vec{r} d x=0 \\
\int_{0}^{L} \delta \vec{b} \cdot\left(\vec{\kappa}-\mathcal{T} \vec{\vartheta}^{\prime}-\vec{d}\right) d x+\int_{0}^{L} \vec{b} \cdot \delta(\vec{\kappa})_{r e l} d x & \\
- & {[\vec{b} \cdot \delta \vec{\vartheta}]_{0}^{L}+\int_{0}^{L} \vec{b}^{\prime} \cdot \delta \vec{\vartheta} d x=0 }
\end{aligned}
$$

By adding equations (17) and (18) to (12), we obtain the modified principle of virtual work in which the variations $\delta(\vec{\gamma})_{r e l}, \delta(\vec{\kappa})_{r e l}, \delta \vec{\vartheta}, \delta \vec{r}, \delta \vec{a}$, and $\delta \vec{b}$ are arbitrary and independent functions and the variations $\delta \vec{r}^{0}, \delta \vec{\vartheta}^{0}, \delta \vec{r}^{L}$, and $\delta \vec{\vartheta}^{L}$ are arbitrary and independent parameters. As all the coefficients at the independent variations must vanish, the following Euler-Lagrange equations of the three-dimensional beam are obtained:

$$
\begin{aligned}
\vec{N}-\vec{a} & =\overrightarrow{0} \\
\vec{M}-\vec{b} & =\overrightarrow{0} \\
\vec{n}+\vec{a}^{\prime} & =\overrightarrow{0} \\
\vec{m}+\vec{b}^{\prime}-\vec{a} \times \vec{r}^{\prime} & =\overrightarrow{0} \\
\vec{\gamma}-\vec{r}^{\prime}-\vec{c} & =\overrightarrow{0} \\
\vec{\kappa}-\mathcal{T} \vec{\vartheta}^{\prime}-\vec{d} & =\overrightarrow{0}
\end{aligned}
$$

along with the boundary conditions

$$
\begin{array}{ll}
\vec{S}^{0}+\vec{a}(0)=\overrightarrow{0} & \vec{S}^{L}-\vec{a}(L)=\overrightarrow{0} \\
\vec{P}^{0}+\vec{b}(0)=\overrightarrow{0} & \vec{P}^{L}-\vec{b}(L)=\overrightarrow{0} .
\end{array}
$$

Equations (19)-(24) constitute the system of six vector equations for six unknown vector functions $\vec{a}(x), \vec{b}(x), \vec{r}(x), \vec{\vartheta}(x), \vec{\gamma}(x)$, and $\vec{\kappa}(x)$ for a given set 
of loads, described by $\vec{n}(x), \vec{m}(x), \vec{S}^{0}, \vec{P}^{0}, \vec{S}^{L}$, and $\vec{P}^{L}$. Equations (23) and (24) are kinematic equations given previously. Equations (21) and (22) are the force and moment equilibrium conditions (see (2)-(3)). The physical meaning of the Lagrangian multipliers $\vec{a}$ and $\vec{b}$ is obvious from (21)-(22): $\vec{a}(x)$ is the cross-sectional force resultant at point $x ; \vec{b}(x)$ is the cross-sectional moment resultant at point $x \cdot \vec{a}(x)$ and $\vec{b}(x)$ satisfy the equilibrium equations and will hence be referred to as the equilibrium force and moment. We have already introduced the cross-sectional force and moment resultants $\vec{N}$ and $\vec{M}$, computed from the strains by the constitutive equations, and these will be termed the constitutive force and moment. Thus, equations (19) and (20) require that the equilibrium force and moment vectors are equal to the constitutive force and moment vectors. These requirements yield the so called 'consistent equilibrium at the cross-section'. Inequality of equilibrium and constitutive stresses is a common characteristic of standard finite element formulations. It may be a substantial source of error of a finite element method, especially in materially non-linear problems.

An application of the consistency conditions in the elastic-plastic finite element analysis of plane frames is presented in the paper by Vratanar and Saje [23].

\section{Governing equations of the beam}

Equations (19)-(24) can be divided into two groups. Equations (19) and (20) are non-linear algebraic equations. Iterative, Newton-like methods are most commonly applied to solve such equations. The remaining equations of the system, (21)-(24), consist of four first order ordinary differential equations. Equations (21)-(23) can be, at least formally, solved when $\vec{n}, \vec{m}, \vec{\gamma}$, and $\vec{\kappa}$ are known functions of $x$ :

$$
\begin{aligned}
& \vec{a}(x)=\vec{a}(0)-\int_{0}^{x} \vec{n}(\xi) d \xi \\
& \vec{b}(x)=\vec{b}(0)+\int_{0}^{x}[\vec{a}(\xi) \times(\vec{\gamma}(\xi)-\vec{c}(\xi))-\vec{m}(\xi)] d \xi \\
& \vec{r}(x)=\vec{r}(0)+\int_{0}^{x}(\vec{\gamma}(\xi)-\vec{c}(\xi)) d \xi .
\end{aligned}
$$

Often the integrated functions are too complicated for the analytical integration to be possible, so the numerical integration must be used. The solution of equation (24) can formally be written in a form of an integral equation

$$
\vec{\vartheta}(x)=\vec{\vartheta}(0)+\int_{0}^{x} \mathcal{T}^{-1}(\vec{\vartheta}(\xi))(\vec{\kappa}(\xi)-\vec{d}(\xi)) d \xi
$$


Again the numerical solution method must be used. Because the unknown function, $\vec{\vartheta}$, appears in the integrand, a different class of numerical methods should be applied, i.e. Runge-Kutta methods for systems of first order ordinary differential equations.

When $\vec{n}(x), \vec{m}(x), \vec{\kappa}(x)$, and $\vec{\gamma}(x)$ are given, unknowns $\vec{a}(x), \vec{b}(x), \vec{r}(x)$, and $\vec{\vartheta}(x)$ can be calculated numerically from (27)-(30). The two remaining unknown functions, $\vec{\kappa}(x)$ and $\vec{\gamma}(x)$, are obtained by the solution of the algebraic equations (19) and (20).

To fulfil the displacement and rotation boundary conditions, strain vectors $\vec{\kappa}(x)$ and $\vec{\gamma}(x)$ must satisfy not only equations (19) and (20), but also equations (29) and (30), evaluated at the right end $(x=L)$. The complete set of the governing equations of the three-dimensional beam then consists of the algebraic equations (19) and (20)

$$
\begin{aligned}
& \vec{N}(x)-\vec{a}(x)=\overrightarrow{0} \\
& \vec{M}(x)-\vec{b}(x)=\overrightarrow{0}
\end{aligned}
$$

the kinematical boundary conditions at $x=L$ for $\vec{r}$ and $\vec{\vartheta}$

$$
\begin{gathered}
\vec{r}(L)-\vec{r}(0)-\int_{0}^{L}[\vec{\gamma}(x)-\vec{c}(x)] d x=\overrightarrow{0} \\
\vec{\vartheta}(L)-\vec{\vartheta}(0)-\int_{0}^{L} \mathcal{T}^{-1}(\vec{\vartheta}(x))(\vec{\kappa}(x)-\vec{d}(x)) d x=\overrightarrow{0},
\end{gathered}
$$

and the statical boundary conditions (25) and (26):

$$
\begin{gathered}
\vec{S}^{0}+\vec{a}(0)=\overrightarrow{0} \\
\vec{P}^{0}+\vec{b}(0)=\overrightarrow{0} \\
\vec{S}^{L}-\vec{a}(0)+\int_{0}^{L} \vec{n}(x) d x=\overrightarrow{0} \\
\vec{P}^{L}-\vec{b}(0)-\int_{0}^{L}[\vec{a}(x) \times(\vec{\gamma}(x)-\vec{c}(x))-\vec{m}(x)] d x=\overrightarrow{0} .
\end{gathered}
$$

Equations (31)-(38) represent an abstract formulation of the governing equations of the three-dimensional beam where the only unknown functions of $x$ are abstract strain vectors $\vec{\gamma}$ and $\vec{\kappa}$. No limitations on the choice of the coordinate basis have been set so far. For each particular vector variable and operator in (31)-(38), we now choose its most suitable basis (we choose between the material or the spatial bases). The componential representation is necessary to formulate the numerical solution of the problem and cannot be 
avoided at this point. An arbitrary vector, $\vec{v}$, can be expressed with respect to either of the two bases

$$
\vec{v}=v_{g 1} \vec{g}_{1}+v_{g 2} \vec{g}_{2}+v_{g 3} \vec{g}_{3}=v_{G 1} \vec{G}_{1}+v_{G 2} \vec{G}_{2}+v_{G 3} \vec{G}_{3} .
$$

The components of the vector, $\left\{v_{g 1}, v_{g 2}, v_{g 3}\right\}$ and $\left\{v_{G 1}, v_{G 2}, v_{G 3}\right\}$, are represented in the matrix form by one-column matrices

$$
\boldsymbol{v}_{g}=\left[\begin{array}{c}
v_{g 1} \\
v_{g 2} \\
v_{g 3}
\end{array}\right], \quad \boldsymbol{v}_{G}=\left[\begin{array}{c}
v_{G 1} \\
v_{G 2} \\
v_{G 3}
\end{array}\right]
$$

The relationship between the two one-column matrices, $\boldsymbol{v}_{g}$ and $\boldsymbol{v}_{G}$, is given by

$$
\boldsymbol{v}_{g}=\mathbf{R} \boldsymbol{v}_{G}
$$

where $\mathbf{R}$ denotes the matrix form of the linear operator $\mathcal{R}$. Thus, the rotation matrix $\mathbf{R}$ does not only represent the rotational operator in the component form, but also the coordinate transformation between the components of a vector with respect to spatial and material bases. An optimal choice of representation seems to be given by Jelenić and Saje [10] and is also used here; equations (31)-(38) then take the matrix form

$$
\begin{gathered}
\mathbf{R} \boldsymbol{N}_{G}(x)-\boldsymbol{a}_{g}(x)=\mathbf{0} \\
\mathbf{R} \boldsymbol{M}_{G}(x)-\boldsymbol{b}_{g}(x)=\mathbf{0} \\
\boldsymbol{r}_{g}^{L}-\boldsymbol{r}_{g}^{0}-\int_{0}^{L} \mathbf{R}\left(\gamma_{G}-\boldsymbol{c}_{G}\right) d x=\mathbf{0} \\
\boldsymbol{\vartheta}_{g}^{L}-\boldsymbol{\vartheta}_{g}^{0}-\int_{0}^{L} \mathbf{T}^{-1}\left(\boldsymbol{\vartheta}_{g}\right) \mathbf{R}\left(\boldsymbol{\kappa}_{G}-\boldsymbol{d}_{G}\right) d x=\mathbf{0} \\
\boldsymbol{S}_{g}^{0}+\boldsymbol{a}_{g}^{0}=\mathbf{0} \\
\boldsymbol{P}_{g}^{0}+\boldsymbol{b}_{g}^{0}=\mathbf{0} \\
\boldsymbol{S}_{g}^{L}-\boldsymbol{a}_{g}^{0}+\int_{0}^{L} \boldsymbol{n}_{g} d x=\mathbf{0} \\
\boldsymbol{P}_{g}^{L}-\boldsymbol{b}_{g}^{0}-\int_{0}^{L}\left[\boldsymbol{a}_{g} \times \mathbf{R}\left(\boldsymbol{\gamma}_{G}-\boldsymbol{c}_{G}\right)-\boldsymbol{m}_{g}\right] d x=\mathbf{0},
\end{gathered}
$$

where the indices $g$ and $G$ indicate the basis used. Componential equations only have sense if components in an equation are expressed with respect to the same basis; that is why the transformation matrix $\mathbf{R}$ is included in some equations. 


\section{Numerical solution of governing equations}

\subsection{Discretization and linearization of equations}

The system of non-linear governing equations (40)-(47) of the beam is solved by Newton's method. However, equations (40) and (41) need to be discretized first. In contrast to the classical Galerkin-type finite element formulation where the integrated weighted residuals are required to vanish, we have chosen the set of discrete points $x_{q}(q=1, \ldots, N)$ and made equations (40)-(41) to be satisfied at these points. After such a collocation-type discretization is made, a set of $2 N$ discrete matrix equations needs to be solved $(q=1, \ldots, N)$ :

$$
\begin{aligned}
& \mathbf{R} \boldsymbol{N}_{G}\left(x_{q}\right)-\boldsymbol{a}_{g}\left(x_{q}\right)=\mathbf{0} \\
& \mathbf{R} \boldsymbol{M}_{G}\left(x_{q}\right)-\boldsymbol{b}_{g}\left(x_{q}\right)=\mathbf{0} .
\end{aligned}
$$

It is further reasonable to discretize the unknown vector functions $\gamma_{G}(x)$ and $\boldsymbol{\kappa}_{G}(x)$. The unknowns $\boldsymbol{\gamma}_{G}(x)$ and $\boldsymbol{\kappa}_{G}(x)$ are replaced by a set of their unknown values at points $x_{p} ; p=1, \ldots, N$, not necessarily equal to the points $x_{q} ; q=1$, $\ldots, N$. The resulting discretized equations constitute a system of $2 N+6$ nonlinear algebraic vector equations of a beam element, where $N$ denotes the number of discretization points. The vector unknowns are $\boldsymbol{r}_{g}^{0}, \boldsymbol{\vartheta}_{g}^{0}, \boldsymbol{a}_{g}^{0}, \boldsymbol{b}_{g}^{0}, \boldsymbol{r}_{g}^{L}$, $\boldsymbol{\vartheta}_{g}^{L}, \boldsymbol{\gamma}_{G}^{p}$, and $\boldsymbol{\kappa}_{G}^{p}(p=1,2, \ldots, N)$.

Note that after the discretization is made, the consistent equilibrium at crosssections is satisfied solely at the chosen discrete points $x_{q}$. The same result could be obtained by the Galerkin-type of discretization if the integration and the interpolation points coincide.

To obtain $\boldsymbol{\gamma}_{G}(x)$ and $\boldsymbol{\kappa}_{G}(x)$ at any $x$, the values of $\boldsymbol{\gamma}$ and $\boldsymbol{\kappa}$ at $x$ are interpolated by

$$
\begin{aligned}
& \gamma_{G}(x)=I_{p}(x) \boldsymbol{\gamma}_{G}^{p} \\
& \boldsymbol{\kappa}_{G}(x)=I_{p}(x) \boldsymbol{\kappa}_{G}^{p}
\end{aligned}
$$

The repeated index $p$ is the summation index over $p=1,2, \ldots, N$. At this point we need to restate that the configuration space of the unknowns is nonlinear because of the multiplicative (non-additive) nature of rotations and the related quantities. Nevertheless, the basic unknown functions $\gamma_{G}(x)$ and $\boldsymbol{\kappa}_{G}(x)$ are additive. This substantially simplifies the Newton update procedure, which will be discussed more precisely in the next subsection, and allows us to introduce the same interpolation functions for the variations of $\gamma_{G}$ and 
$\kappa_{G}$

$$
\begin{aligned}
& \delta \boldsymbol{\gamma}_{G}(x)=I_{p}(x) \delta \boldsymbol{\gamma}_{G}^{p} \\
& \delta \boldsymbol{\kappa}_{G}(x)=I_{p}(x) \delta \boldsymbol{\kappa}_{G}^{p} .
\end{aligned}
$$

Making use of (52) and (53) the Jacobian matrix of the system of discrete equations (48)-(49) and (42)-(47) is easily obtained and its derivation will therefore not be presented here. We do need to discuss some critical points, though. The linearization is made in the sense of the first variation of functional. The independent (primary) unknowns are $\boldsymbol{r}_{g}^{0}, \boldsymbol{\vartheta}_{g}^{0}, \boldsymbol{a}_{g}^{0}, \boldsymbol{b}_{g}^{0}, \boldsymbol{r}_{g}^{L}, \boldsymbol{\vartheta}_{g}^{L}, \boldsymbol{\gamma}_{G}^{p}$, and $\boldsymbol{\kappa}_{G}^{p}(p=1,2, \ldots, N)$. Therefore we need to express the variations of any other vector as a function of the unknowns and their variations: $\delta \boldsymbol{r}_{g}^{0}, \delta \boldsymbol{\vartheta}_{g}^{0}, \delta \boldsymbol{a}_{g}^{0}$, $\delta \boldsymbol{b}_{g}^{0}, \delta \boldsymbol{r}_{g}^{L}, \delta \boldsymbol{\vartheta}_{g}^{L}, \delta \boldsymbol{\gamma}_{G}^{p}$, and $\delta \boldsymbol{\kappa}_{G}^{p}(p=1,2, \ldots, N)$. The variations of (27)-(29) are easily obtained. The variation of the rotational vector as the function of the variations of primary unknowns follows from (6), when expressed with respect to the selected basis for $\boldsymbol{\kappa}$ and $\boldsymbol{\vartheta}$ :

$$
\mathbf{R} \delta \boldsymbol{\kappa}_{G}=\delta \boldsymbol{\vartheta}_{g}^{\prime}
$$

Inserting (53) into (54) and integrating gives the relation between the variation of the rotational vector and the variations of the unknowns

$$
\delta \boldsymbol{\vartheta}_{g}(x)=\delta \boldsymbol{\vartheta}_{g}^{0}+\left(\int_{0}^{x} I_{p} \mathbf{R}(\xi) d \xi\right) \delta \boldsymbol{\kappa}_{G}^{p}
$$

We need to be more attentive to the variation of rotation matrix $\mathbf{R}$ and to the variation of constitutive equations (10)-(11). Since the derivation of the variation of the rotation matrix has been widely discussed (see, e.g. [9], [3], [7]), only the final result is shown here

$$
\delta \mathbf{R}=\delta \boldsymbol{\Theta} \mathbf{R}
$$

where $\delta \boldsymbol{\Theta}$ denotes the skew-symmetric matrix, whose axial vector is the variation of the rotational vector $\delta \boldsymbol{\vartheta}_{g}$. If we write $\delta \boldsymbol{\vartheta}_{g}=\left[\begin{array}{c}\delta \vartheta_{1} \\ \delta \vartheta_{2} \\ \delta \vartheta_{3}\end{array}\right]$, then

$$
\delta \boldsymbol{\Theta}=\left[\begin{array}{ccc}
0 & -\delta \vartheta_{3} & \delta \vartheta_{2} \\
\delta \vartheta_{3} & 0 & -\delta \vartheta_{1} \\
-\delta \vartheta_{2} & \delta \vartheta_{1} & 0
\end{array}\right]
$$

The variation of rotation matrix (56) can be further expressed by the variation of strain vector $\boldsymbol{\kappa}_{G}$, when considering equation (55). 
The linearization of the constitutive equations gives

$$
\begin{aligned}
\delta \boldsymbol{N}_{G} & =\delta \mathcal{C}_{N}=\mathbf{C}_{\gamma \gamma} \delta \gamma_{G}+\mathbf{C}_{\gamma \kappa} \delta \kappa_{G} \\
\delta \boldsymbol{M}_{G} & =\delta \mathcal{C}_{M}=\mathbf{C}_{\kappa \gamma} \delta \gamma_{G}+\mathbf{C}_{\kappa \kappa} \delta \kappa_{G}
\end{aligned}
$$

where the components of the matrices $\mathbf{C}_{\gamma \gamma}, \mathbf{C}_{\gamma \kappa}, \mathbf{C}_{\kappa \gamma}$, and $\mathbf{C}_{\kappa \kappa}$ are the directional derivatives of $\mathcal{C}_{N}$ and $\mathcal{C}_{M}$ with respect to $\boldsymbol{\gamma}_{G}(x)$ and $\boldsymbol{\kappa}_{G}(x)$.

\subsection{Newton's iteration method}

In applying Newton's method to the problem, the non-linearity of the configuration space of the beam requires a special care. The variations of the unknowns are elements of its tangent space. In order to obtain the unknowns, we need to apply an update procedure which maps the unknowns from the tangent to the configuration space. Following Newton's iteration scheme, a system of linear equations is solved at each iteration step $n=0,1,2, \ldots$

$$
\mathbf{K}^{[n]} \delta \boldsymbol{y}=-\boldsymbol{f}^{[n]},
$$

where $\mathbf{K}^{[n]}$ is the global Jacobian tangent matrix, $\boldsymbol{f}^{[n]}$ is the residual vector of equations (48)-(49) and (42)-(47), both in iteration $n$, and $\delta \boldsymbol{y}$ is a vector of corrections, which, in classical Newton's method in linear vector spaces, is added to the previous solution iterate vector $\boldsymbol{y}^{[n]}$. The non-linearity of the configuration space, on the other hand, requires a non-classical update procedure. Its basic idea is illustrated in Figure 3.

The non-linear configuration space is symbolically illustrated by a curved surface. The iterative approximation to the solution vector, $\boldsymbol{y}$, is an element of the non-linear space. The correction of the solution vector, $\delta \boldsymbol{y}$, is an element of a linear space, here symbolically illustrated with a tangent plane. A new approximation of the solution is obtained, first by determining new solution vector, point $\boldsymbol{y}+\delta \boldsymbol{y}$ on the tangent plane, and then by projecting the point onto the configuration space.

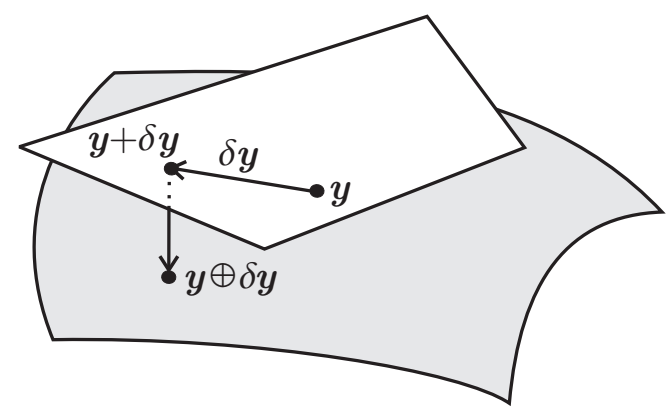

Fig. 3. The update procedure in a non-linear configuration space. 
As a result of an iteration step, the corrections of the unknowns, $\delta \boldsymbol{r}^{0}, \delta \boldsymbol{\vartheta}^{0}$, $\delta \boldsymbol{a}^{0}, \delta \boldsymbol{b}^{0}, \delta \boldsymbol{r}^{L}, \delta \boldsymbol{\vartheta}^{L}, \delta \boldsymbol{\gamma}_{G}^{p}$, and $\delta \boldsymbol{\kappa}_{G}^{p}$, are obtained. The position vector and stress resultants are linear quantities. Therefore, their corrections are added to the iterative values:

$$
\begin{array}{ll}
\boldsymbol{r}_{g}^{0[n+1]}=\boldsymbol{r}_{g}^{0[n]}+\delta \boldsymbol{r}_{g}^{0} & \boldsymbol{r}_{g}^{L[n+1]}=\boldsymbol{r}_{g}^{L[n]}+\delta \boldsymbol{r}_{g}^{L} \\
\boldsymbol{a}_{g}^{0[n+1]}=\boldsymbol{a}_{g}^{0[n]}+\delta \boldsymbol{a}_{g}^{0} & \boldsymbol{b}_{g}^{0[n+1]}=\boldsymbol{b}_{g}^{0[n]}+\delta \boldsymbol{b}_{g}^{0} .
\end{array}
$$

Thanks to the suitable choice of the basis for the strain vectors, also the corrections of strains can be added to the iterative values

$$
\begin{gathered}
\boldsymbol{\gamma}_{G}^{p[n+1]}=\boldsymbol{\gamma}_{G}^{p[n]}+\delta \boldsymbol{\gamma}_{G}^{p} \\
\boldsymbol{\kappa}_{G}^{p[n+1]}=\boldsymbol{\kappa}_{G}^{p[n]}+\delta \boldsymbol{\kappa}_{G}^{p} .
\end{gathered}
$$

The proof for the strain vector $\boldsymbol{\kappa}_{G}$ is not obvious and is based on the additivity of the angular velocity vector, when expressed with respect to the material basis (see, e.g. Shabana [16]).

The boundary rotations are, of course, not additive. Their update goes in a standard way. First, corrections of boundary rotation matrices, $\delta \mathbf{R}^{0}, \delta \mathbf{R}^{L}$ are calculated from $\delta \boldsymbol{\vartheta}^{0}$ and $\delta \boldsymbol{\vartheta}^{L}$ by the Rodrigues formula (1). Then new boundary rotation matrices are obtained by matrix multiplication:

$$
\begin{aligned}
& \mathbf{R}^{0[n+1]}=\delta \mathbf{R}^{0} \mathbf{R}^{0[n]} \\
& \mathbf{R}^{L[n+1]}=\delta \mathbf{R}^{L} \mathbf{R}^{L[n]} .
\end{aligned}
$$

Finally, the Spurrier algorithm [20] is used to extract new boundary rotational vectors $\boldsymbol{\vartheta}^{0[n+1]}$ and $\boldsymbol{\vartheta}^{L[n+1]}$ from $\mathbf{R}^{0[n+1]}$ and $\mathbf{R}^{L[n+1]}$.

The update procedure just described is thus almost completely additive. Such an update demands less numerical operations and is more suitable from the theoretical and numerical point of view. When the update is additive, any ordinary additive type of interpolation can be applied in (50) and (51), and the objectivity of this additive update is not questionable. Unfortunately the additive update may lead to the equation (43) not being precisely satisfied. This error may contribute to the global error of the element. When we wish to use a more precise element, a different update procedure for curvatures is suggested, here termed the 'non-additive update'. It stems from the variations of rotations, which are consistent with the variations of the boundary rotations. Rewriting (54) with respect to the material basis we get

$$
\delta \boldsymbol{\kappa}_{G}=\delta \boldsymbol{\vartheta}_{G}^{\prime}
$$

The solution of (62), i.e. function $\delta \boldsymbol{\vartheta}_{G}(x)$, is the variation of rotational vector, expressed with respect to the material basis, which is fully consistent with $\delta \boldsymbol{\vartheta}_{g}^{L}$ at $x=L$. Due to the non-linearity of the configuration space, $\delta \boldsymbol{\vartheta}_{G}$ is 
not the same as when $\delta \boldsymbol{\vartheta}_{G}$ is chosen as the basic incremental quantity. The corresponding increment of strain vector $\boldsymbol{\kappa}_{G}$ is then obtained by transforming the variation $\delta \boldsymbol{\kappa}_{G}$ with respect to the variation of rotational vector

$$
\Delta \boldsymbol{\kappa}_{G}=\mathbf{T}^{T}\left(\delta \boldsymbol{\vartheta}_{G}\right) \delta \boldsymbol{\kappa}_{G}
$$

and added to the current iterative value

$$
\kappa_{G}^{[n+1]}=\boldsymbol{\kappa}_{G}^{[n]}+\Delta \boldsymbol{\kappa}_{G} .
$$

Note that the objectivity of strain measures is preserved even with the "nonadditive update', which is the consequence of the direct interpolation of strains and of the addition of increment in equation (64).

\section{Numerical examples}

We will present several numerical examples in order to demonstrate the performance and accuracy of the proposed formulation. To enable the comparison with other formulations, a linear elastic material is employed in all numerical examples. The operators $\mathcal{C}_{N}$ and $\mathcal{C}_{M}$ in (10)-(11) are taken to be diagonal

$\boldsymbol{N}_{G}=\left[\begin{array}{ccc}E A_{1} & 0 & 0 \\ 0 & G A_{2} & 0 \\ 0 & 0 & G A_{3}\end{array}\right]\left(\boldsymbol{\gamma}_{G}-\boldsymbol{\gamma}_{G, 0}\right) \quad \boldsymbol{M}_{G}=\left[\begin{array}{ccc}G J_{1} & 0 & 0 \\ 0 & E J_{2} & 0 \\ 0 & 0 & E J_{3}\end{array}\right]\left(\boldsymbol{\kappa}_{G}-\boldsymbol{\kappa}_{G, 0}\right)$.

Here $E$ and $G$ denote elastic and shear moduli of material; $A_{1}$ is the crosssectional area; $J_{1}$ is the torsional inertial moment of the cross-section; $A_{2}$ and $A_{3}$ are the shear areas in the principal inertial directions $\vec{G}_{2}$ and $\vec{G}_{3}$ of the cross-section; $J_{2}$ and $J_{3}$ are the cross-sectional inertial moments about its principal directions $\vec{G}_{2}$ and $\vec{G}_{3}$.

Finite elements with various number of interpolation points (taken to coincide with the collocation points) are used in order to investigate the influence of their number on the accuracy of numerical solutions. A member of the family of the finite elements described above is uniquely determined by its number of interpolation points. A particular element is marked by the symbol ' $E$ ' and equipped with the subscript describing the number, $N$, of interpolation points used. Element $E_{N}$ has thus $6 N+18$ degrees of freedom (according to Equations (48)-(49) and (42)-(47)). Since the nodal values of strains and the internal force and moment at the left boundary are eliminated at the element level, each element has finally 12 external degrees of freedom. $E_{2}$ with two interpolation points was the element of the lowest order used. We wish to 
stress that the order of interpolation functions is not limited to any particular value in our computer program, so elements of any order can be chosen.

The quadratic convergence of Newton's method was achieved in all numerical examples. The iteration was terminated when the Euclidean norm of the vector of nodal unknowns, $\|\delta \boldsymbol{y}\|_{2}$, and of the vector of unbalanced residual forces, $\|\boldsymbol{f}\|_{2}$, was less than $10^{-9}$.

\subsection{Lateral buckling of a right-angle frame}

In this classical problem, first introduced by Argyris et al. [2] and studied by many authors (e.g. [10], [12], [18]), we analyze a simply-supported thin rectangular right-angle frame. The undeformed line of centroids lies in the $X Y$-plane. The support at one end allows the translation in the $X$-direction and the rotation about the $Z$-direction. The support on the other end allows only the rotation about the $Z$-direction. The frame is subjected to the in-plane moments, as shown in Figure 4.

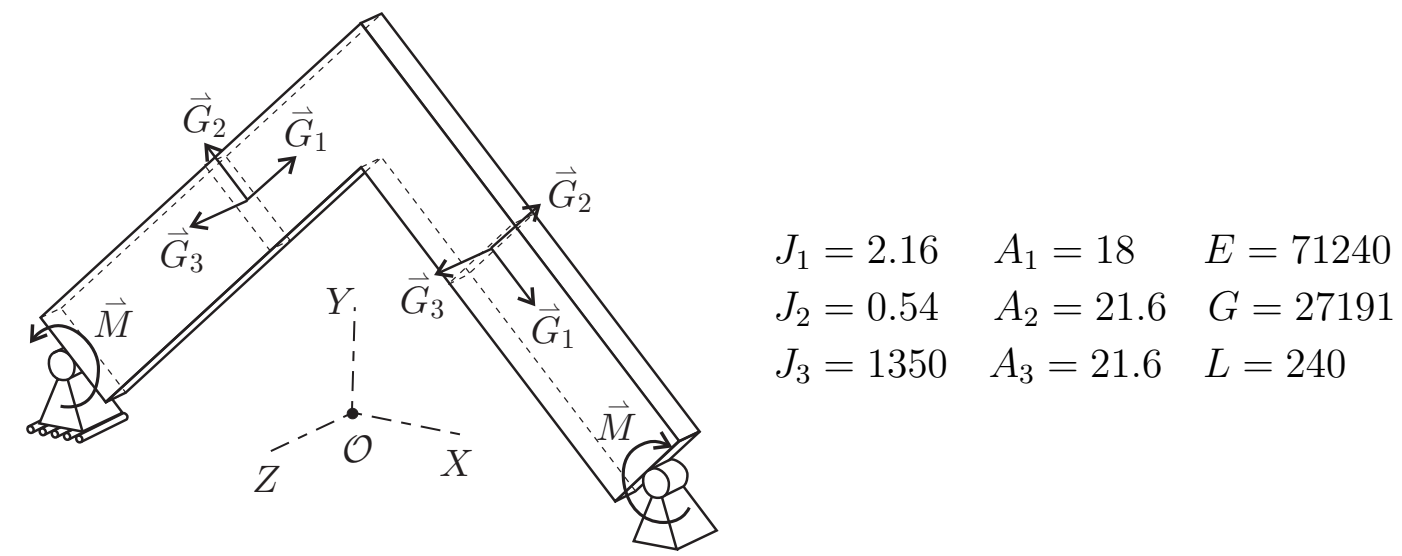

Fig. 4. Lateral buckling of right-angle frame.

Due to the extreme slenderness of the rectangular cross-section (the thickness to height ratio is taken to be $1 / 50$ ), the frame buckles out of the plane. This occurs when the tangent stiffness matrix becomes singular. The iterative bisection-based algorithm to find the critical moment was used. In an each step of the bisection algorithm, the iterative value for the critical moment is applied in a single load step. Only two Newton's iterations per step were needed. The out-of-plane buckling moment $M_{c}$ is sought by the use of different-order elements and several finite element meshes. Our results are shown in Table 1. The comparisons with the analytical solution provided by Timoshenko and Gere [22] and various numerical solutions by other authors are also presented.

The four-digit accurate critical moment was obtained either by a single element with 6 interpolation points (the total number of degrees of freedom is 54) or 
Table 1

Critical moment $M_{c}$ of the simply supported right-angle frame.

\begin{tabular}{|c|c|c|c|c|c|c|c|c|c|}
\hline$E$ & e.d.o.f. & d.o.f. & $n_{e}=1$ & $n_{e}=2$ & $n_{e}=3$ & $n_{e}=4$ & $n_{e}=5$ & $n_{e}=6$ & $n_{e}=10$ \\
\hline$E_{2}$ & & & \pm 685.99 & \pm 626.88 & \pm 623.20 & \pm 622.54 & \pm 622.35 & \pm 622.29 & \\
\hline$E_{3}$ & 24 & 126 & \pm 626.31 & \pm 622.31 & $\pm 622.23^{*}$ & \pm 622.22 & & & \\
\hline$E_{4}$ & 18 & 78 & \pm 622.40 & $\pm 622.22^{*}$ & \pm 622.22 & & & & \\
\hline$E_{5}$ & 12 & 48 & $\pm 622.23^{*}$ & \pm 622.22 & & & & & \\
\hline$E_{6}$ & 12 & 54 & $\pm 622.22^{*}$ & & & & & & \\
\hline$E_{8}$ & & & \pm 622.22 & & & & & & \\
\hline [10] & 12 & 27 & \pm 622.2 & & & & & & \\
\hline$[12]$ & & 66 & & & & & & & \pm 626.7 \\
\hline$[18]$ & 126 & 126 & & & & & & & \pm 626 \\
\hline analy & tical soly & tion & \pm 622.21 & & & & & & \\
\hline
\end{tabular}

by the mesh of 3 elements with 3 interpolation points (totally 108 degrees of freedom). From Table 1 one can see a rapid convergence of the numerical solution with the increasing number of interpolation point. The comparison with other authors shows that only the solution by Jelenić and Saje [10] gives the results of equal precision. In the present formulation the total number of the degrees of freedom per an element is larger than in [10], because two vector unknowns, $\boldsymbol{\gamma}_{G}$ and $\boldsymbol{\kappa}_{G}$, are interpolated in our present formulation. The interpolation degree of each vector suffices to be one degree smaller than in [10] to achieve an equal precision. Note that the two formulations have the same number of external degrees of freedom.

Thus, the concept of interpolating of both strain vectors increases indeed the total number of internal degrees of freedom. As these are condensed on an element level, the number of external degrees of freedom per element still remains 12. We believe that such a strain-based formulation has advantages in materially non-linear problems, such as in the localization of strains in concrete structures due to the softening of material, in which the cost of additional degrees of freedom is compensated by a better robustness and a higher accuracy of the solution.

The advantage of the present approach is further demonstrated by its enhanced numerical stability, which results also in obtaining the numerical solutions much closer to the analytical ones. The analytical solution by Timoshenko and Gere [22] is based on the assumption of inextensibility, shear and in-plane bending rigidities of the beam which are not very well approximated by the data in Figure 4 taken from the literature. We do not know the reason of so being; maybe the researchers experienced difficulties or were even unable to handle the case if larger values for $G A_{2}, G A_{3}, E A_{1}$, and $E J_{3}$ were assumed. 
Table 2

Critical moment $M_{c}$ of the inextensibile, shear and in-plane stiff simply supported right-angle frame.

\begin{tabular}{lrrrrrr}
\hline$E$ & $n_{e}=1$ & $n_{e}=2$ & $n_{e}=3$ & $n_{e}=4$ & $n_{e}=5$ & $n_{e}=6$ \\
\hline$E_{2}$ & \pm 686.08462 & 626.87586 & 623.18912 & 622.52749 & 622.35490 & 622.27385 \\
$E_{3}$ & 626.30670 & 622.29411 & 622.12423 & 622.21134 & 622.21030 & 622.21005 \\
$E_{4}$ & 622.38305 & 622.21077 & 622.20993 & 622.20992 & & \\
$E_{5}$ & 622.21450 & 622.20993 & & & & \\
$E_{6}$ & 622.21000 & & & & & \\
$E_{8}$ & 622.20993 & & & & & \\
\multicolumn{1}{l}{ analytical solution } & $\pm \mathbf{6 2 2 . 2 0 9 9 2}$
\end{tabular}

$E=$ type of element, $n_{e}=$ number of elements

To show robustness of the present method, we now approximate the inextensibility, shear and in-plane bending rigidities by taking much larger values for $G A_{2}, G A_{3}, E A_{1}$, and $E J_{3}$

$$
E A_{1}=G A_{2}=G A_{3}=E J_{3}=10^{15}
$$

and keeping the remaining data as they were $\left(E J_{2}=38469.6, G J_{3}=58732.56\right.$, $L=240$ ). As shown in Table 2 , we were able to obtain the results accurate to 7 digits by the use of one higher-order element or, equivalently, several low-order elements. Although the problem so modified becomes highly numerically sensitive, which resulted in a slightly slower convergence of the Newton's method, the large rigidities did not substantially affect the accuracy of the results.

In this numerical example, both update procedures give the same results, because we have not been dealing with the out-of-plane rotations. From that point of view the forthcoming examples are more interesting.

\subsection{Buckling of a cantilever right-angle frame}

The right-angle frame is now clamped at one end and subjected to the point forces at its free end, as shown in Figure 5(a). Force $F$ in the $X$-direction is ascendant with the largest value taken to be 1.485 . To obtain the post- 
buckling behaviour, a small perturbation force $F_{p}=0.001 F$ in the out-ofplane direction is applied. We used a rather large number of load steps (100) to make the graph of the load-displacement response being drawn smoothly. The final deformed centroidal axis is shown in Figure 5(b) together with its projection on the $X Z$-plane.
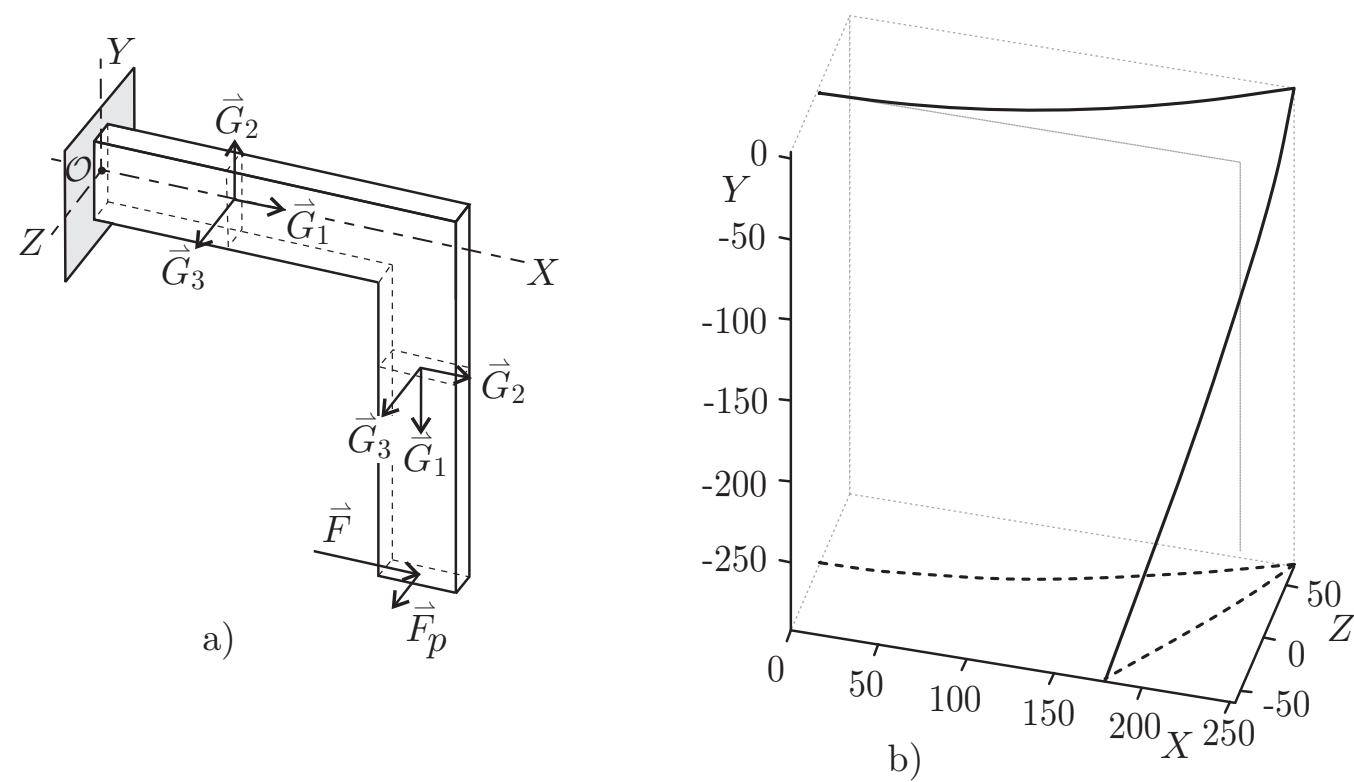

Fig. 5. Lateral buckling of a cantilever right-angle frame: the initial configuration (a), and the final deformed centroidal axis (b).

The results were obtained by using both update procedures, and the forcedisplacement diagrams are compared in Figure 6. The two graphs almost agree. The results in Figure 6 were obtained by the mesh of 12 elements with 5 interpolation points per element and are in agreement with the results of other finite-element formulations [4], [18], [19].

When the additive update for curvatures (Equation (61)) is employed, the equation (43) for the rotational vector at $x=L$

$$
\boldsymbol{\vartheta}_{g}^{L}=\boldsymbol{\vartheta}_{g}^{0}-\int_{0}^{L} \mathbf{T}^{-1}\left(\boldsymbol{\vartheta}_{g}\right) \mathbf{R}\left(\boldsymbol{\kappa}_{G}-\boldsymbol{d}_{G}\right) d x
$$

may not give the same value for the right-boundary rotation $\boldsymbol{\vartheta}_{g}^{L}$ as the direct update (see the discussion in Section 6.2). In Table 3 we display the relative error

$$
\frac{\left\|\boldsymbol{\vartheta}_{g}^{L}-\boldsymbol{\vartheta}_{g}^{0}+\int_{0}^{L} \mathbf{T}^{-1}\left(\boldsymbol{\vartheta}_{g}\right) \mathbf{R}\left(\boldsymbol{\kappa}_{G}-\boldsymbol{d}_{G}\right) d x\right\|_{2}}{\left\|\boldsymbol{\vartheta}_{g}^{L}\right\|_{2}}
$$

for each element in the mesh. The error is considerable and spans from roughly $10^{-3}$ to $10^{-5}$. Yet it pactically does not influence the results for the remaining 


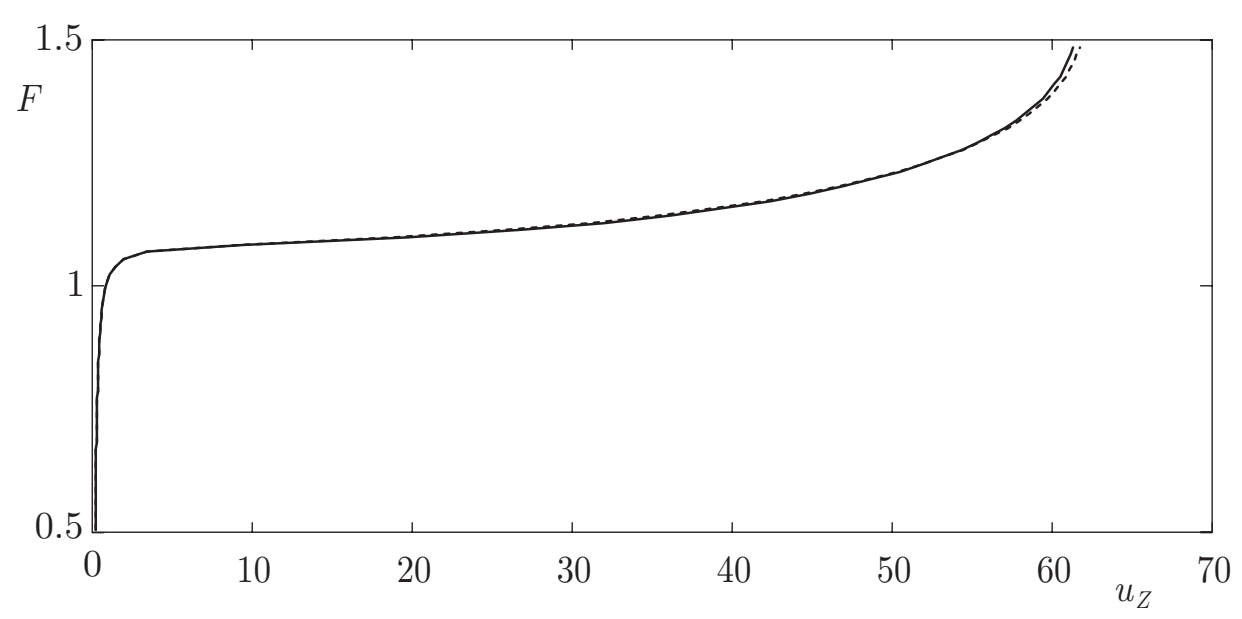

Fig. 6. Out-of-plane displacement versus in-plane force diagram (additive update dashed line, non-additive update - solid line).

variables. Note also that this error vanishes completely if the non-additive update for curvatures (Equations (63) and (64)) is used instead.

Table 3

Relative error of the right-boundary rotation in the finite element mesh.

\begin{tabular}{|c|c|c|c|c|c|c|c|c|c|c|c|c|}
\hline e. & 1 & 2 & 3 & 4 & 5 & 6 & 7 & 8 & 9 & 10 & 11 & 12 \\
\hline$\varepsilon$ & $8 \cdot 10^{-5}$ & $3 \cdot 10^{-4}$ & $5 \cdot 10^{-4}$ & $7 \cdot 10^{-4}$ & $9 \cdot 10^{-4}$ & $1 \cdot 10^{-3}$ & $1 \cdot 10^{-3}$ & $1 \cdot 10^{-3}$ & $9 \cdot 10^{-4}$ & $7 \cdot 10^{-4}$ & $4 \cdot 10^{-4}$ & $2 \cdot 10^{-4}$ \\
\hline
\end{tabular}

\subsection{Cantilever, bent to a helical form}

We consider a very interesting example, presented by Ibrahimbegovic [9]. When a straight in-plane cantilever is subjected to a point moment at its free end, it deforms into a part of a circle, which results in a pure bending of the cantilever. A much more interesting behaviour is observed when an outof-plane point force is added at the free end of the cantilever (Figure 7(a)). The out-of-plane force causes the out-of-plane displacements of the beam. We took the same geometric and material properties of the cantilever as in [9]:

$$
\begin{aligned}
G A_{2} & =G A_{3}=E A_{1}=10^{4} \quad L=10 \\
E J_{2} & =E J_{3}=G J_{1}=10^{2} .
\end{aligned}
$$




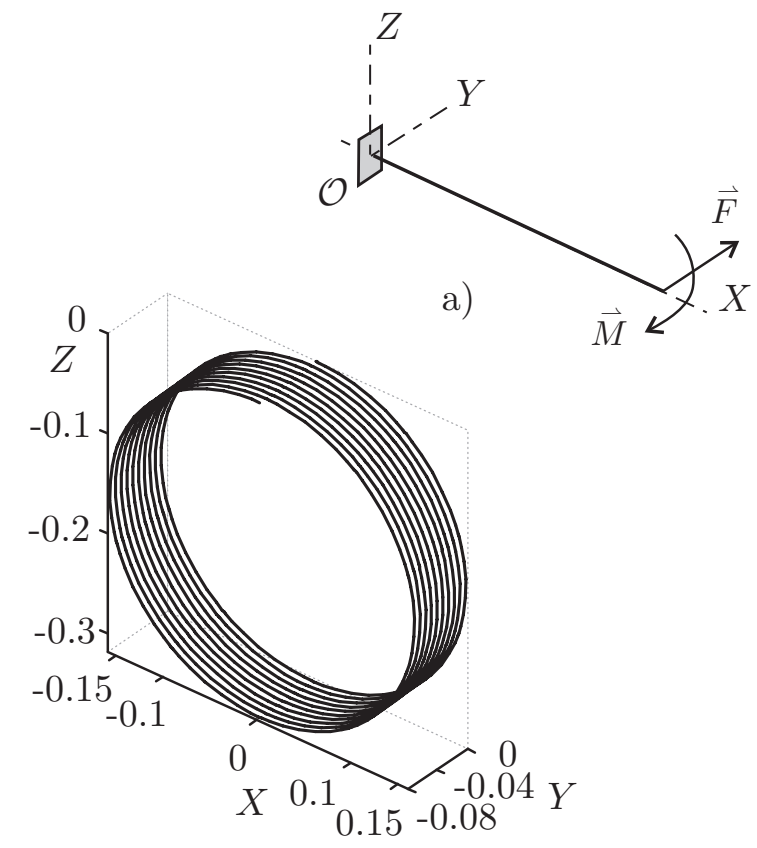

b)

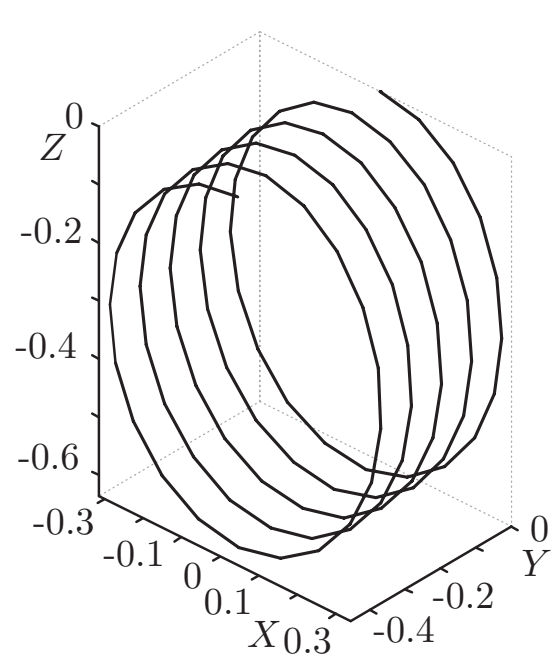

c)

Fig. 7. Illustration of the undeformed beam together with the loading (a) and the deformed axis of centroids of the beam (b), (c).

The two loads $M=200 \pi \lambda$ and $F=50 \lambda$ were increased incrementally from $\lambda=0$ to $\lambda=1$ in 1000 steps. The beam was modelled by a mesh of 5 elements with 8 interpolation points, and the more precise non-additive update was used. The result of a simultaneous application of moment and force is a beam, bent into a helical form (Figure 7(b)). For the numerical values of geometrical and material properties taken, the beam bends into ten circles, which do not lie in the same plane, yet very close together. The maximum out-of-plane displacement is roughly -0.08 . It must be emphasized that at the final load stage the beam is bent in the opposite direction to the applied force.

In the second example, where the cross-section was not doubly symmetric, we took $E J_{2}=2 \cdot 10^{2} \neq E J_{3}=10^{2}$ while the remaining data were the same as in the previous example. In principle, similar behaviour is obtained, though considerably different quantitative results are observed. The final deformed shape is shown in Figure 7(c). The influence of a different shape of the crosssection is evident. The circles are bigger (approximately twice as big as in the former case) and the number of circles is lower (only 5 circles constitute the final shape). Finally, the maximum out-of-plane displacement of the beam is - surprisingly - substantially larger. It must be stressed that, in both cases, the beam is bent in the opposite direction to the applied force.

The displacement $u_{Y}$ of the free end of the cantilever as a function of loading factor $\lambda$ is shown in Figure 8. Observe that for the doubly symmetric cross-section (the solid line), the curve, describing the displacement, oscillates around the zero value with its amplitude slowly decreasing. Thus, during the 


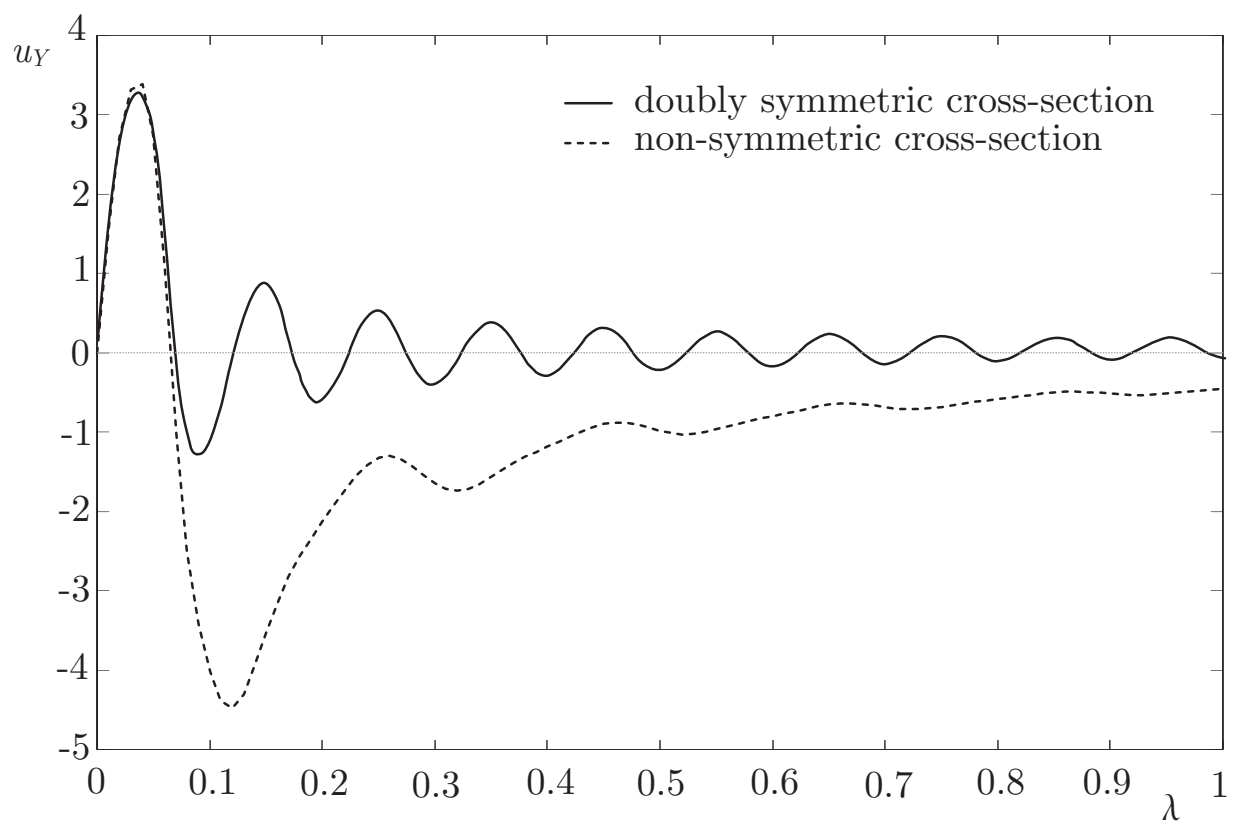

Fig. 8. Free end displacement, $u_{Y}$, versus loading factor, $\lambda$.

load increase, the beam bends on different sides of the $X Z$-plane. From Figure 8 it is also clear that the behaviour of the beam with the non-symmetric crosssection (the dashed line) is different in comparison to the doubly-symmetric case.

The analyses of Ibrahimbegovic [9] and later by Battini and Pacoste [4] show the importance of the suitable parametrization of rotations in order to obtain the correct results. The parametrization of rotations in the present formulation avoids problems of large rotations. The values of the free-end displacements for the doubly symmetric cross-section $\left(E J_{2}=E J_{3}=10^{2}\right)$ almost completely agree with the results in [9] and [4]. The comparisons for the non-symmetric cross-sections beams $\left(E J_{2}=2 \cdot 10^{2} \neq E J_{3}=10^{2}\right)$ were not possible as they are, to our best knowledge, not available in literature.

\subsection{Bending of a pre-twisted cantilever}

In the last example, we study cantilevers with a pretwisted cross-section. The beams are clamped at one end and loaded as in the previous example. The centroidal axis is straight at the beginning, but the cross-sections are twisted about the centroidal axis. The initial twist along the length of the beam is described by the rotation angle as a linear function of arc-length $x$. We took the same geometric and material properties as in the previous case (with the 
non-symmetric cross-section)

$$
\begin{aligned}
G A_{2} & =G A_{3}=E A_{1}=10^{4}, \quad L=10 \\
E J_{3} & =G J_{1}=10^{2}, \quad E J_{2}=2 \cdot 10^{2} .
\end{aligned}
$$

Different angles of the pretwist are analyzed. Firstly, the beam is linearly twisted up to the $2 \pi$ (twist at the free end). The twist is applied first (Figure $9(\mathrm{a})$ ) in the positive direction, and then (Figure $9(\mathrm{~b})$ ) in the opposite direction. For a discussion on twisted beams see the paper by Zupan and Saje [25].

The results are completely different from those in Figure 7(c). The pretwist of the beam strongly enhances the resistance to bending, compared to the non-twisted case. In the positive direction pretwist case (Figure $9(\mathrm{a})$ ), once the value $\lambda=0.455$ was reached, the shape of the beam practically did not change any more. The same phenomenon was observed in the second case for the value $\lambda=0.315$.

By increasing the pretwist angle, a similar behaviour is expected. We studied $10 \pi$ pretwisted beam. The final shape is shown in Figure 10(a). The shapes of the deformed beam at smaller load steps were more like the helical form. After the increase of load, the influence of the pretwist became evident. The resemblance to the behaviour of a straight beam is more evident from the loaddisplacement diagram (Figure 10(b)), where some similarity to results in Figure 8 (the solid line) can be observed. The amplitudes of the free-end displacements are quantitatively similar, while the displacements of the pretwisted beam oscillate about the slanted line (and not about the zero value).
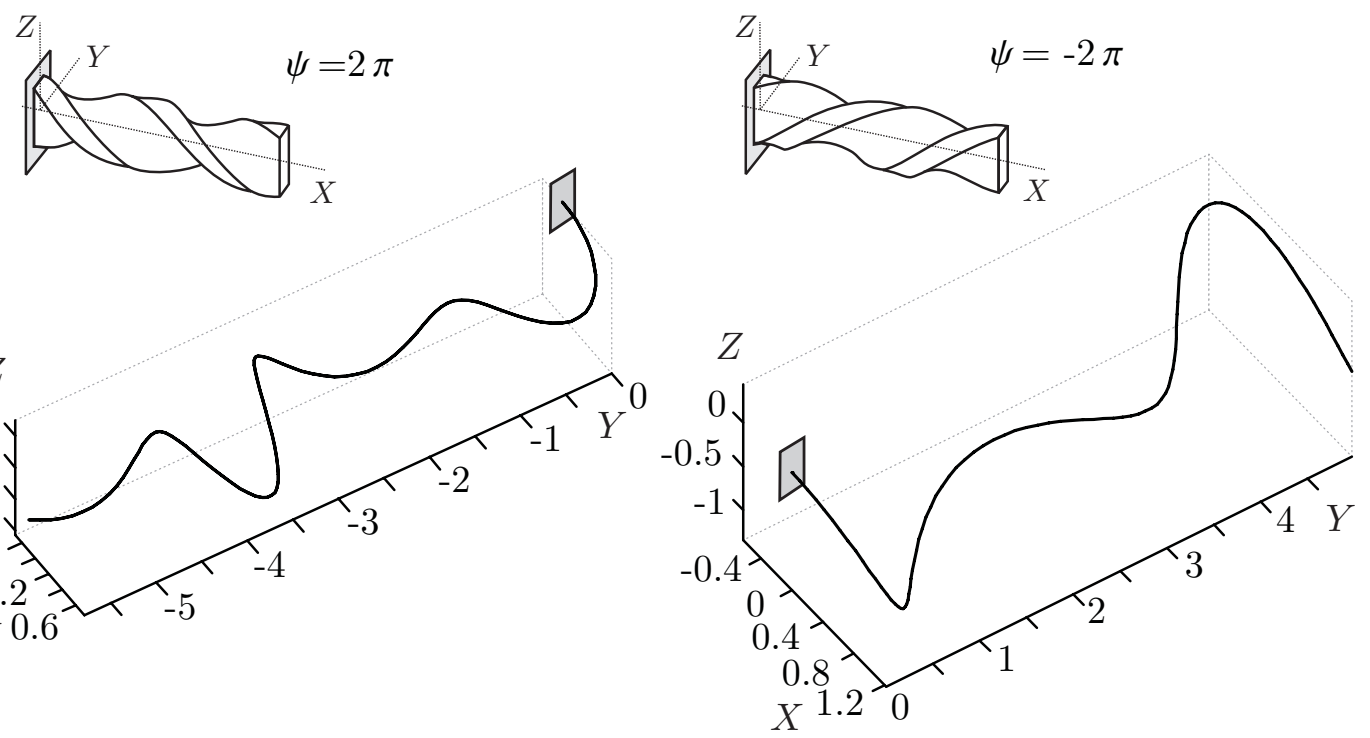

a)

b)

Fig. 9. The deformed axis of centroids of $2 \pi$-pretwisted beams. 


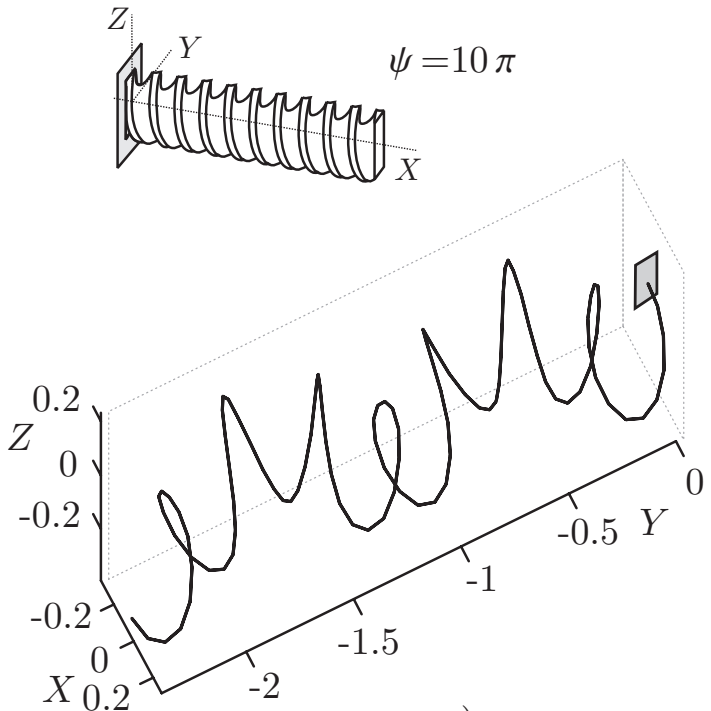

a)

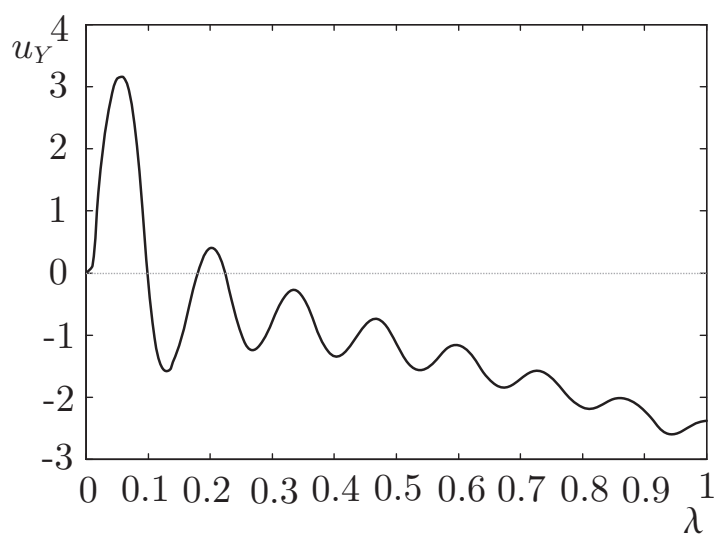

b)

Fig. 10. Deformed axis of centroids of $10 \pi$-pretwisted beam (a) and its free-end displacement in the direction of the force versus loading factor (b).

This test proves that the present formulation takes the initially non-planar configuration of the beam into account properly even when we deal with extremely large rotations. For futher references about twisted beams, see e.g. [11] and [25].

\section{Conclusions}

We presented a new finite element formulation of the geometrically exact threedimensional beam theory, based on the interpolation of strain vectors. The essential points of the formulation are:

(i) A modified principle of virtual work is proposed in which the strain vectors are the only unknown functions. It follows that the strain vectors are the only functions that need to be interpolated. The formulation based on strain interpolation is inherently strain-objective and has many advantages in materially non-linear problems, such as in the analysis of localization of concrete structures due to softening of material.

(ii) Displacements and rotational vectors (or their variations) are not interpolated.

(iii) The interpolation of both strain vectors increases the total number of internal degrees of freedom, while the number of external degrees of freedom remains 12.

(iv) The consistency condition that the equilibrium and the constitutive internal force and moment vectors are equal, is enforced to be satisfied at the given 
points (the 'collocation'). This considerably improves the accuracy of the internal forces and moments at the points, which is particularly important in materially non-linear problems.

(v) The determination of internal forces and moments does not require the differentiation. It then follows that the accuracy of the internal forces and moments is of the same order as the accuracy of the basic variables. This is an important advantage compared to formulations where the derivatives are needed for the evaluation of internal forces.

(vi) The present finite elements are free of locking.

(vii) A number of finite elements of different order have been tested by various numerical examples. A rapid convergence has been the characteristic of all elements.

\section{Acknowledgment}

This work was supported by the Ministry of Education, Science and Sport of the Republic of Slovenia through the grant S2-792-016/19121. The support is gratefully acknowledged.

\section{References}

[1] J. H. Argyris, "An excursion into large rotations", Comput. Methods Appl. Mech. Eng. 32, 85-155, 1982.

[2] J. H. Argyris, P. C. Dunne, G. Malejannakis, D. W. Scharpf, "On large displacements - small strain analysis of structures with rotational degrees of freedom", Comput. Methods Appl. Mech. Eng. 14, 99-135, 1978.

[3] S. N. Atluri, A. Cazzani, "Rotations in computational solid mechanics", Arch. Comput. Methods in Eng. 2, 49-138, 1995.

[4] J. Battini, C. Pacoste, "Co-rotational beam elements with warping effects in instability problems", Comp. Methods Appl. Mech. Eng. 191, 1755-1789, 2002.

[5] M. A. Crisfield, "A consistent co-rotational formulation for non-linear, threedimensional beam elements", Comp. Meth. Appl. Mech. Engng. 81, 131-150, 1990.

[6] M. A. Crisfield, G. Jelenić, "Objectivity of strain measures in the geometrically exact three-dimensional beam theory and its finite-element implementation", Proc. Roy. Soc. London A 455, 1125-1147, 1999.

[7] M. Géradin, D. Rixen, "Parametrization of finite rotations in computational dynamics: a review", Revue européenne des éléments finis 4, 497-553, 1995.

[8] A. Ibrahimbegovic, "On the finite element implementation of geometrically nonlinear Reissner's beam theory: 3d curved beam element", Comput. Methods Appl. Mech. Eng. 122, 11-26, 1995. 
[9] A. Ibrahimbegovic, "On the choice of finite rotation parameters", Comput. Methods Appl. Mech. Eng. 149, 49-71, 1997.

[10] G. Jelenić, M. Saje, "A kinematically exact space finite strain beam model-finite element formulation by generalized virtual work principle", Comput. Methods Appl. Mech. Eng. 120, 131-161, 1995.

[11] R. H. MacNeal, R. L. Harder, "A proposed standard set of problems to test finite element accuracy", Finite Elem. Anal. Design 1, 3-20, 1985.

[12] B. Nour-Omid, C. C. Rankin, "Finite rotation analysis and consistent linearization using projectors", Comput. Methods Appl. Mech. Eng. 93, 353384, 1991.

[13] I. Planinc, M. Saje, B. Čas, "On the local stability condition in the planar beam finite element", Structural Engineering and Mechanics 12(5), 507-526, 2001.

[14] E. Reissner, "On finite deformation of space-curved beams", J. Appl. Math. Phys. 32, 734-744, 1981.

[15] M. Schulz, F. C. Filippou, "Non-linear spatial Timoshenko beam element with curvature interpolation", Int. J. Numer. Methods Eng. 50, 761-785, 2001.

[16] A. A. Shabana, "Dynamics of Multibody Systems". Second Edition. Cambridge University Press, 1998.

[17] J. C. Simo, "A finite strain beam formulation. The three-dimensional dynamic problem. Part I", Comput. Methods Appl. Mech. Eng. 49, 55-70, 1985.

[18] J. C. Simo, L. Vu-Quoc, "A three-dimensional finite-strain rod model. Part II: Computational aspects", Comput. Methods Appl. Mech. Eng. 58, 79-116, 1986.

[19] W. M. Smoleński, "Statically and kinematically exact nonlinear theory of rods and its numerical verification", Comput. Methods Appl. Mech. Eng. 178, 89113, 1999.

[20] R. A. Spurrier, "Comment on Singularity-free extraction of a quaternion from a direction-cosine matrix", J. Spacecraft 15, 255, 1978.

[21] B. Tabarrok, M. Farshad, H. Yi, "Finite element formulation of spatially curved and twisted rods", Comput. Methods Appl. Mech. Eng. 70, 275-299, 1988.

[22] S. P. Timoshenko, J. M. Gere, "Theory of Elastic Stability", McGraw-Hill, New York, 1961.

[23] B. Vratanar, M. Saje, "A consistent equilibrium in a cross-section of an elasticplastic beam", Int. J. Solids Structures 36, 311-337, 1999.

[24] D. Zupan, M. Saje, "A new finite element formulation of three-dimensional beam theory based on interpolation of curvature", CMES: Computer Modeling in Engineering and Sciences 4, No. 2, 301-318, 2003.

[25] D. Zupan, M. Saje, "On "A proposed standard set of problems to test finite element accuracy": The twisted beam", Finite Elem. Anal. Design (to be published). 